\title{
sciforum
}

\section{Evolutionary variations in HLH domain modulates the fast inactivation phase in calcium selective TRP channels.}

\author{
Lisandra Flores-Aldama ${ }^{a, b}$, Kattina Zavala ${ }^{c}$, Juan C Opazo ${ }^{c}$, Daniel Bustos ${ }^{d}$, Wendy \\ Gonzalez, $^{\text {d,e }}$, Sebastian Brauchi ${ }^{\text {a,e }}$ \\ ${ }^{a}$ Instituto de Fisiología, Facultad de Medicina, Universidad Austral de Chile \\ ${ }^{b}$ Escuela de Graduados, Facultad de Ciencias, Universidad Austral de Chile \\ ${ }^{c}$ Instituto de Ciencias Ambientales y Evolutivas, Facultad de Ciencias, Universidad Austral de Chile \\ ${ }^{d}$ Center for Bioinformatics and Molecular Simulations (CBSM), Universidad de Talca. \\ ${ }^{e}$ Millennium Nucleus of Ion Channels-associated Diseases (MiNICAD)
}

\section{Graphical Abstract}

Insert grafical abstract figure here

\begin{abstract}
.
TRPV5 and TRPV6 are highly calciumselective channels from the Transient Receptor Potential (TRP) family ${ }^{1}$. These channels are considered gatekeepers of epithelial calcium transport, essential for calcium homeostasis ${ }^{1}$. At negative potentials, the channels exhibit a twophase calcium-dependent inactivation where the slow component is shared and determined by the binding of $\mathrm{Ca}^{2+}$-Calmodulin complex to the $\mathrm{C}$ terminal region of the channel ${ }^{(2,3)}$. In contrast, the rapid phase of inactivation is independent of the calcium-Calmodulin complex and allows differentiating both channels from a functional point of view; while TRPV6 shows a very robust inactivation, at the same calcium concentrations, the inactivation of TRPV5 conductance is modest ${ }^{4}$. The intracellular loop $\mathrm{S} 2-\mathrm{S}^{5}$ and residues downstream the transmembrane segment $\mathrm{S6}^{6}$ has been associated to the differences observed in the kinetics of the rapid phase of inactivation. However, the exact location of the putative calcium-binding site and the molecular mechanism governing this process
\end{abstract}


are not known. A thorough phylogenetic reconstruction in vertebrates suggest that the genes encoding for these channels duplicates more than once during evolution, naturally introducing the same set of mutations within a HLH domain located at the $\mathrm{N}$ terminal region. Further sequence analysis unveiled that the HLH domain acts as a fingerprinting in both channels. Molecular dynamics simulations, allowed us to identify a putative calcium-binding site that put together three different portions of the folded channel. By means of site-directed mutagenesis and patch clamp electrophysiology we reversed the phenotype of inactivation in these channels, confirming that the HLH sequence serves as modulator for the calcium-induced inactivation process. We conclude that subtle evolutionaryrelated variations within the binding region affect the phenotype of the fast inactivation phase.

\section{Introduction (optional)}

Materials and Methods (optional)

Results and Discussion (optional)

Conclusions (optional)

\section{References (mandatory)}

1. $\quad$ Dekker et al., Cell Calcium 33 (2003) :497-507.

2. De Groot et al., Mol. and Cell. Biol. 31 (2011): 2845-2853

3. $\quad$ Derler et al., J Physiol 577.1 (2006): 31-44

4. Hoenderop et al., Journal of Physiology 537.3 (2001): 747-761

5. $\quad$ Niulius et al.; J.Biol. Chem. (2002) 277:30852-30858

6. Suzuki et al., Biom. Bioph R.C. (2002) 91:278-285 